\title{
ASSESSMENT OF LEFT ATRIAL FUNCTION IN PATIENTS WITH MITRAL VALVE DISEASES
}

\author{
ALPHONSA MATHEW ${ }^{1}$, RANJAN SHETTY ${ }^{2}$, KRISHNANANDA N ${ }^{3}$, NAVIN PATIL ${ }^{4}$, KARTHIK RAO N $^{5 *}$, \\ JYOTHI SAMANTH ${ }^{3}$, BALAJI $0^{4}$, DIPANJAN B ${ }^{4}$, RAHUL KOTIAN ${ }^{6}$
}

\begin{abstract}
${ }^{1}$ Department of Adult ECHO Lab, Narayana Institute of Cardiac Sciences, Bengaluru, Karnataka, India. ${ }^{2}$ Department of Cardiology, Manipal Hospital, Bengaluru, Karnataka, India. ${ }^{3}$ Department of Cardiovascular Technology, SOAHS, Manipal University, Manipal, Karnataka, India. ${ }^{4}$ Department of Pharmacology, KMC, Manipal University, Manipal, Karnataka, India. ${ }^{5}$ Department of Medicine, KMC, Manipal University, Manipal, Karnataka, India. ${ }^{6}$ Department of Medical Imaging Technology, SOAHS, Manipal University, Manipal, Karnataka, India. Email: karthikrao85@gmail.com
\end{abstract}

Received: 12 August 2016, Revised and Accepted: 17 August 2016

\section{ABSTRACT}

Objective: To observe and assess left atrial (LA) function by observing the differences in conventional Doppler echo parameters of left ventricular inflow and LA appendage among patients with mitral valve disease.

Methods: A total of 43 mitral valve disease patients appearing consecutively for echocardiogram (ECHO) test at the cardiology department in a tertiary care hospital were recruited into the study as per the pre-set inclusion and exclusion criteria. The data from the ECHO were pooled using Microsoft Excel and analyzed using SPSS software by application of appropriate statistical tests.

Results: Of the 43 objects, 39 had multiple sclerosis (MS), 3 had magnetic resonance (MR), and 2 of them were found to have both MS and MR. The major presenting symptom as observed in 33 patients was dyspnea. LA maximum volume was found to be $91 \pm 59 \mathrm{ml}$ and minimum was $66 \pm 51 \mathrm{ml}$. LA expansion index was $128 \pm 91$. LA active emptying fraction was $29 \pm 13$, and passive emptying fraction was $31 \pm 15$. No significant change in LA global strain among groups with MR and without MR was observed. Further, no significant difference was observed in LA indices such as LA emptying fraction, LA passive emptying fraction, atrial fraction, and LA expansion index among groups having MR and no MR.

Conclusion: LA contractile, reservoir, and conduit function were significantly reduced in mitral valve diseases due to increased hemodynamic load. No significant difference was noted in global LA strain irrespective of MS or MR.

Keywords: Valvular disease, Left atria, Strain, Contractile function.

(c) 2016 The Authors. Published by Innovare Academic Sciences Pvt Ltd. This is an open access article under the CC BY license (http://creativecommons. org/licenses/by/4. 0/) DOI: http://dx.doi.org/10.22159/ajpcr.2016.v9i6.14677

\section{INTRODUCTION}

Atrial function, in a close interdependence with left ventricular (LV) function, plays a key role in maintaining an optimal cardiac performance. The left atrial (LA) modulates LV filling its reservoir, conduit, and booster pump function, whereas LV function influences LA function throughout the cardiac cycle. The LA can act to increase LA pressure (in significant atrial disease) and can react to increased LV filling pressure (in significant ventricular disease). LA remodeling is related to LV remodeling, and LA function has a central role in maintaining optimal cardiac output despite impaired LV relaxation and reduced LV compliance [1]. Understanding, how each component of LA function is influenced by LV performance and how each LA phasic function contributes to maintain an optimal stroke volume in normal and diseased hearts, is important for interpreting data derived from quantification of LA function. During LV systole and isovolumic relaxation, the LA functions as a reservoir, receiving blood from the pulmonary veins, and storing energy in the form of pressure. This atrial function is modulated by LV contraction, through the descent of the LV base during systole, by right ventricular systolic pressure transmitted through the pulmonary circulation and by LA properties (i.e. relaxation and chamber stiffness). During early LV diastole and diastasis, the LA functions as a conduit. Blood is transferred into the LV through the LA via a small pressure gradient during early diastole and flows passively from the pulmonary veins into the LV during diastasis. The conduit function is modulated especially by $\mathrm{LV}$ diastolic properties (LV relaxation and early diastolic pressures). During late LV diastole, the LA functions as a pump, the LA contraction augmenting LV stroke volume by approximately $20-30 \%$ in normal participants and substantially more in the presence of impaired LV relaxation. LA booster pump function is modulated by LV compliance, LV end-diastolic pressure, and LA intrinsic contractility. It has been demonstrated that the FrankStarling mechanism is also operative in the LA. LA output increases as atrial diameter increases, which contributes to maintaining a normal stroke volume. Moreover, LA contractile function might decrease in the presence of severe LA dilation when the optimal Frank-Starling relationship is exceeded. Thus, the study of LA function can provide additional information, incremental to LA volume measurement. To achieve a better understanding of LA function, knowledge of the LA pressure and volume changes during the cardiac cycle is important. Beginning with mitral valve closure, blood flows into the LA through the pulmonary veins, producing an increase in volume accompanied by a continuous pressure rise ("v" wave). After the opening of the mitral valve, atrial volume begins to decrease accompanied by a parallel fall in LA pressure. During atrial diastasis, the volume remains relatively constant and atrial pressure increases. Atrial volume starts to decrease with the beginning of active atrial emptying, accompanied by a new pressure rise (" $\mathrm{A}$ " wave). The evaluation of volume-pressure curves is the most accurate and representative index for characterizing LA mechanical function in different hemodynamic conditions [2]. On the other hand, echocardiography is a simple and widely available tool that has been increasingly used for the non-invasive assessment of LA function [3-8]. This may include several parameters ranging from conventional methods based on the pulse wave (PW) Doppler evaluation of transmitral flow, pulmonary venous flow, and calculation of LA phasic volumes, to newer methods based on measurements of 
atrial myocardial velocities or deformation. LA function is influenced by changes in LA afterload. The latter is increased in mitral stenosis as a result of increased resistance to blood flow imposed by the stenotic mitral valve. However, to date, there are no data about these indices for LA in multiple sclerosis (MS) patients. Recently, atrial S/Sr obtained from the tissue Doppler imaging (TDI) has been applied to regional LA function assessment [9-12]. Hence, we hypothesized present study to observe LA function parameters and assessment of LA function by noting the differences in conventional Doppler echo parameters of LV inflow and LA appendage among patients with mitral valve disease and also observing the changes occurring in LA deformation among patients with mitral valve disease.

\section{METHODS}

The study was approved by the Institutional Ethics Committee Hospital, Manipal, Karnataka, India. A study population of 43 consecutive patients with significant mitral valve diseases who appeared for echocardiographic test and who met the following inclusion criteria were recruited into our study:

1. Mitral regurgitation of at least moderate grade

2. Mitral stenosis of at least moderate grade

3. Combined significant mitral stenosis and regurgitation.

\section{Exclusion criteria}

It includes:

1. LV systolic dysfunction, ejection fraction $<50 \%$

2. Ischemic heart disease

3. Aortic valve diseases more than mild degree (atrial strain [AS] or aortic regurgitation)

4. Cardiomyopathies

5. Pericardial diseases

6. Systemic disorders

7. Prosthetic valve.

Sample size was calculated using $80 \%$ power and $5 \%$ level of significance. Hence, calculated sample size was $n=42$ cases. The site of the study was the Department of Cardiology, KMC, Manipal. Materials needed includes echocardiography data. An echocardiogram was done using vivid 7-echocardiography system (GE) and with a $2.5 \mathrm{MHz}$ transducer. The same are stored. Microsoft Excel and SPSS software were needed for data pooling and statistical analysis. This protocol was approved by the Institutional Review Board, and informed consent was obtained from all patients. The study was conducted according to the principles of the Declaration of Helsinki.

PW Doppler-derived peak A wave velocity and A wave time velocity integral (TVI) of mitral inflow can reflect LA booster pump function. Atrial fraction calculated as the ratio between the TVI of the A wave and the TVI of diastolic transmitral flow, representing the percentage of ventricular filling during atrial contraction, may also be used as a measure of LA contractile function.

\section{Atrial fraction=TVI A wave/TVI mitral inflow}

The main limitations of these parameters are their dependence on heart rate and loading conditions. Systolic (S), diastolic (D), and atrial reversal (Ar) waves, measured by PW Doppler at the level of pulmonary venous flow, are determined by events that regulate phasic LA pressure and can theoretically describe the reservoir, conduit, and booster pump function of the LA. These parameters are highly dependent on LV diastolic properties. Thus, an increase in duration of the A wave reflects rather an increase in LV end-diastolic pressure than an increase in atrial performance, thus limiting the usefulness of this parameter as a measure of LA pump function. Atrial ejection force, representing the force exerted by the LA to propel blood across the mitral valve into the LV during atrial systole, is another parameter proposed to describe the atrial mechanical function. It can be calculated as the product of the mass and acceleration of blood passing through the mitral annulus during the accelerative phase of atrial systole:

Atrial ejection force=mass*acceleration

Mass is further defined as the product of the density of blood $\left(1.06 \mathrm{~g} / \mathrm{cm}^{3}\right)$ and the volume of blood passing through the mitral orifice during this portion of atrial ejection. Assuming that the upslope of the A wave is nearly flat and A wave acceleration is relatively constant, the above formula becomes:

Atrial ejection force $=0.531 * 1.063^{*}$ mitral annulus area*peak A velocity 2

The robustness, reproducibility, and incremental value of this parameter over LA volume assessment have not been documented. The most common method used for the assessment of LA function is based on the measurement of LA phasic volumes: Maximum volume (Volmax) measured just before the opening of the mitral valve, minimal volume (Volmin) measured at the closure of the mitral valve, and the volume just before the atrial contraction measured at the onset of the $\mathrm{P}$ wave on the ECG (Vol P). The following three indices, reflecting the phasic functions of the LA (reservoir, conduit, and booster pump) can be derived from the LA phasic volume:

\section{LA expansion index $=($ Volmax-Volmin $) /$ Volmin $* 100$}

LA passive emptying fraction $=($ Volmax - Vol P $) /$ Volmax $* 100$

LA active emptying fraction $=($ VolP-Volmin $) /$ Vol $P * 100$

Published reference values for maximum and minimum LA volumes are $22-6 \mathrm{ml} / \mathrm{m}^{2} 22$ and $9-4 \mathrm{ml} / \mathrm{m}^{2} 23$, respectively.

The biplane area-length method or the biplane Simpson's method is currently recommended for the measurement of LA volumes by twodimensional (2D) echocardiography. However, in future, it is likely that three-dimensional (3D) echocardiography, providing more accurate and reproducible measurements, will emerge as the best and preferred method to calculate LA volumes. The main limitation of LA phasic volume derived parameters is the inability to distinguish between the increase in LA function due to a larger amount of blood received/ejected and a real increase in intrinsic LA compliance/contractility.

\section{TDI parameters}

TDI allows the quantification of LV longitudinal myocardial velocities, providing a relatively load independent measure of both systolic and diastolic LV function. Several studies have demonstrated that peak velocity of the mitral annulus in late diastole a', secondary to atrial contraction, can be used as a marker of atrial function. Unlike the early diastolic tissue Doppler velocities, late diastolic septal and lateral velocities (a') seem not to be significantly different.

TDI can also be used for the assessment of regional LA function. The velocities recorded at the level of atrial segments adjacent to the mitral annulus are higher than the velocities of the superior segments of the atrium that are relatively fixed. However, the velocities are influenced by translation and tethering, and therefore, are not able to distinguish true atrial contraction from mitral annular and ventricular motion. In contrast, AS and strain rate demonstrate a good site specificity and are able to describe the longitudinal shortening and lengthening of the atrium which are discordant with ventricular longitudinal motion. Moreover, strain rate analysis, with a good temporal resolution, allows the quantification of all three components of LA function which are systolic AS rate for reservoir function, early diastolic AS rate for conduit function, and late diastolic AS rate for contractile function [4]. In addition, TDI allows non-invasive quantification of atrial electromechanical delay, by measurement of the time interval from the onset of the $\mathrm{P}$ wave on the ECG to the peak of A wave on the atrial myocardial velocities curves [4]. In normal participants, the electromechanical coupling is 
shortest at the level of the right atrial wall, and progressively longer at the level of the interatrial septum, and the lateral LA wall. Thus, total atrial conduction time can be non-invasively estimated as the time from the beginning of the P wave on the ECG to the peak of A wave velocity recorded at the level of the lateral LA wall [13]. Although this time interval overestimates the atrial electromechanical delay, it has demonstrated a very good correlation with total atrial conduction time measured by signal-averaged ECG technique, and thus can be a suitable parameter for the identification of a potential atrial substrate vulnerable for atrial fibrillation. TDI is an angle dependent technique and wall-by-wall sampling is time-consuming, limiting the use of this method in clinical practice $[4,13]$.

\section{Speckle tracking echocardiography (STE) parameters}

STE is a new technique based on tracking the movement of natural acoustic markers (speckles) present on standard grayscale images. STEderived strain and strain rate parameters are relatively independent of wall tethering and loading conditions. STE has been recently proposed for the quantification of atrial function [14] allowing a comprehensive, angle independent assessment of myocardial deformation, and overcoming the limitations of TDI. Global longitudinal LA strain and strain rate parameters, such as the average deformation of all LA segments recorded in one view, can be detected.

\section{RESULTS}

A total of 43 consecutive patients, 30 females and 12 males (Fig. 1), with significant mitral valve diseases meeting the inclusion criteria who appeared for echocardiographic test were recruited into our study. Of the 43 patients, 39 had MS, 3 had magnetic resonance (MR), and 2 of them were found to have both MS and MR (Fig 2a and b).

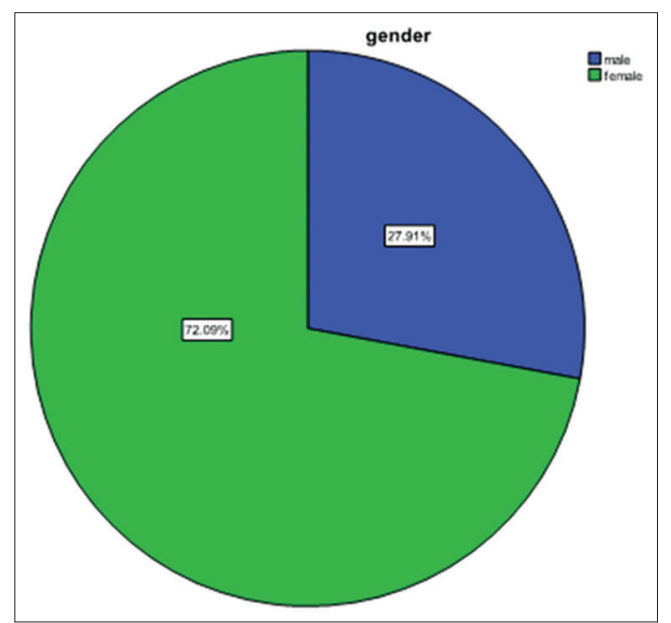

Fig. 1: Gender distribution of participants
Among the study population, the major presenting symptom as observed in 33 patients was dyspnea. 8 of them complained of fatigue, 10 had history of paroxysmal nocturnal dyspnea, and 11 had orthopnea, and $\mathrm{AF}$ was found in 1 patient (Fig. 3a-e)

The 2D parameters of aorta and LA are shown in (Table 1). Doppler echocardiographic features of mitral valve are shown in (Table 2). The mean mitral valve peak velocity was found to be $2.4 \pm 0.5 \mathrm{~m} / \mathrm{s}$, and mean peak A wave velocity was $1.9 \pm 0.6 \mathrm{~m} / \mathrm{s}$. The atrial fraction is found to be 1.16. The mean electromechanical delay was $538 \pm 55 \mathrm{~m}$ seconds

Volumetric parameters of LA are shown in Table 3. LA volmax was found to be $91 \pm 59 \mathrm{ml}$; LA minimum volume was $66 \pm 51 \mathrm{ml}$. LA expansion index, which is an indirect index of LA reservoir function, was $128 \pm 91$. LA active emptying fraction, which is an index of contractile function, was $29 \pm 13$ and passive emptying fraction was $31 \pm 15$; this is nothing but the measure of LA conduit function.

LA function indices are shown in Table 4. Atrial ejection force, which is a measure of the contractile function of LA, was found to be 9.32 in patients who are having mitral valve diseases. LA global strain is shown in Table 5. The value of global strain is found to be 7.4 in patients who met inclusion criteria of the study with the minimum value of 0.2 and the maximum value of 25 . It was found that there is no significant change in LA global strain among groups with MR and without MR (Table 6). There was no significant difference in LA indices such as LA emptying fraction, LA passive emptying fraction, atrial fraction, and LA expansion index among groups having MR and no MR (Table 7).

\section{DISCUSSION}

In the study conducted by Mi-Seung Shin et al., about echocardiographic assessments of LA strain and volume in healthy participants and patients with mitral valvular heart disease by TDI and 3D echocardiography, it was found that the maximal LA volume index of MS and MR patients were $57.6 \pm 18.5$ and $56.8 \pm 17.1$, respectively. LA active emptying fraction was lower in the MS and MR, which were 19.2 \pm 10.2 and $23.2 \pm 9.8$, respectively. The mean LA filling velocity was greater in the MR patients than in MS patients. The value of global strain was found to be 7.4 in patients who met inclusion criteria of the study with the minimum value of 0.2 and maximum value of 25 .

In the study conducted by Mi-Seung Shin et al., it was found that in case of mitral stenosis, the circumferential and radial tissue velocities were significantly lower than the longitudinal tissue velocities. The maximal and minimal LA volumes were negatively correlated with the LA active emptying fraction $(\mathrm{r}=-0.45$ and $\mathrm{r}=-0.68$, respectively; $\mathrm{p}<0.05)$ and positively correlated with the LA filling velocity $(r=0.63$ and $r=0.41$, respectively; $\mathrm{p}<0.05$ ).

The maximal LA volume was positively correlated with the active emptying velocity $(\mathrm{r}=0.50, \mathrm{p}=0.02)$ and negatively correlated with

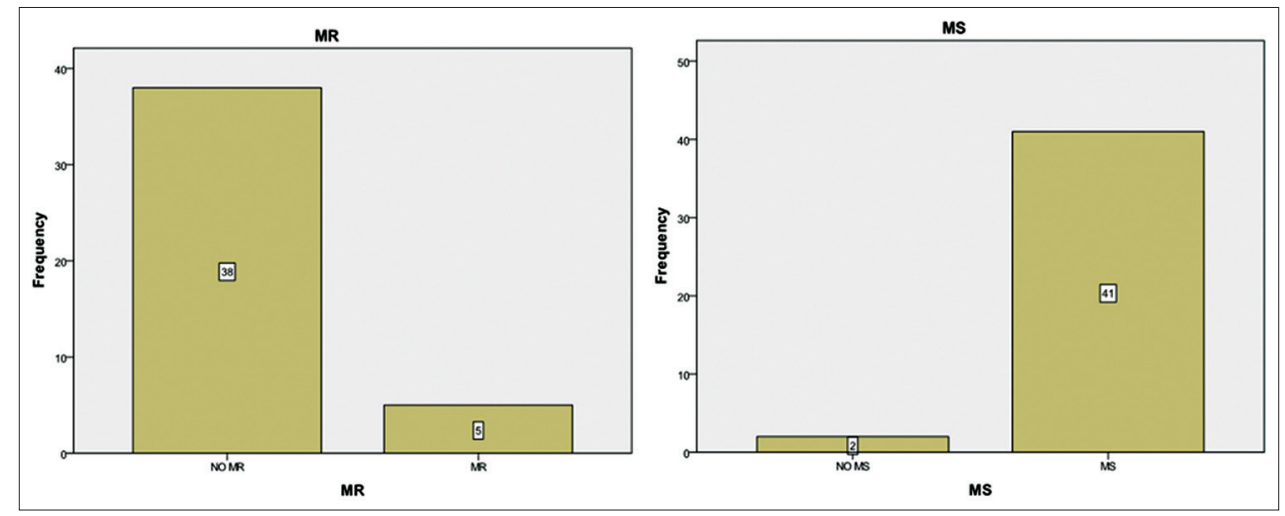

Fig. 2: Pathological distribution of mitral valve diseases 


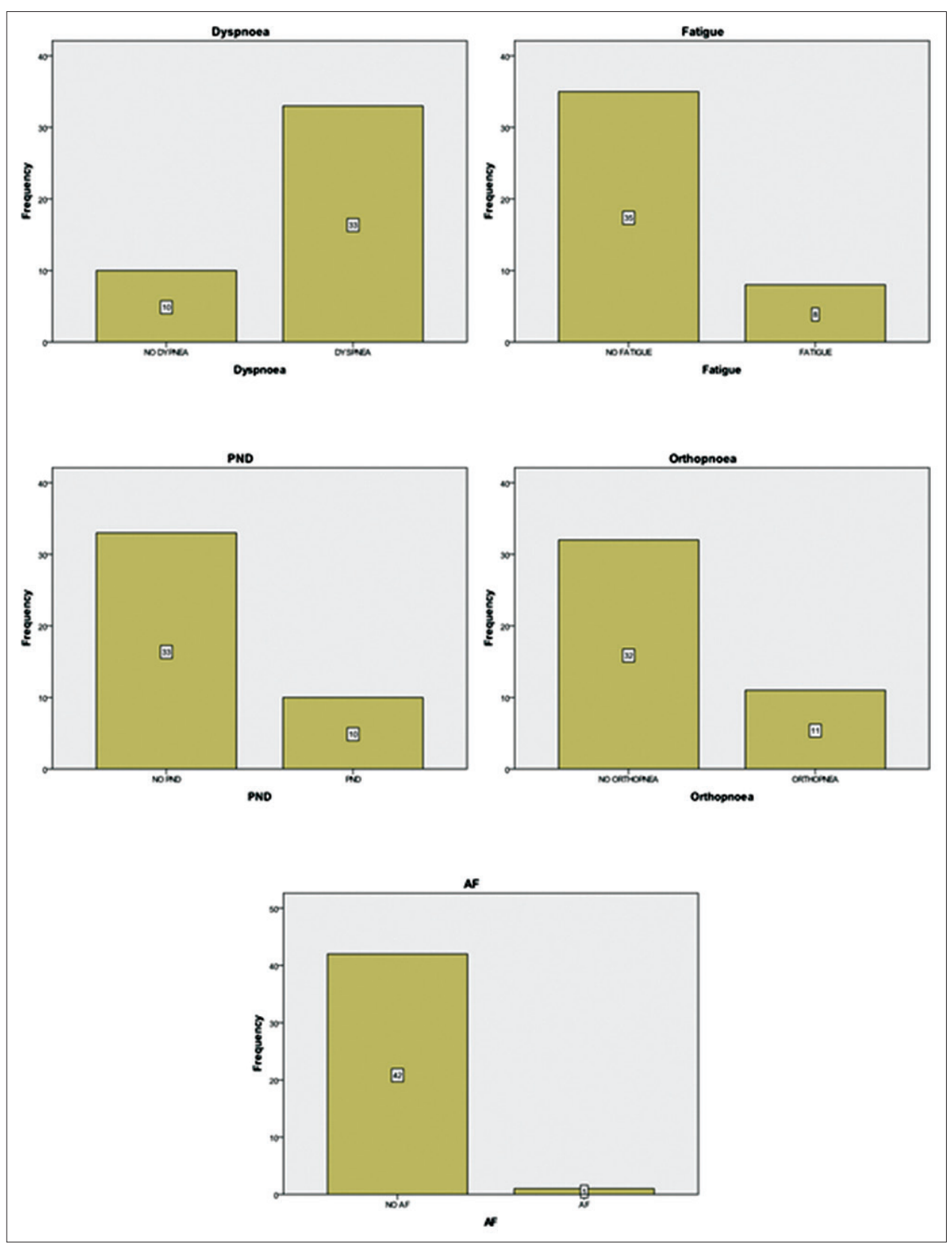

Fig. 3: Clinical features of patients with mitral valve disease

Table 1: 2D parameters of aorta and LA

\begin{tabular}{lllll}
\hline Parameters & AO_ROOT & LA_AP & LA_AO ratio & LA_IS \\
\hline N (valid) & 43 & 43 & 43 & 43 \\
Mean & 26.95 & 46.79 & 1.78 & 62.63 \\
Standard deviation & 3.80 & 8.03 & 0.37 & 9.04 \\
\hline
\end{tabular}

AO: Aorta, LA: Left Atrium, AP: Anterio-posterior, IS: Infero-superior, 2D: Two-dimensional

the radial peak systolic tissue velocity $(\mathrm{r}=-0.53, \mathrm{p}=0.02)$ but was not significantly related to the longitudinal and circumferential peak systolic tissue velocities. The maximal LA volume was negatively correlated with the posterior wall peak longitudinal late diastolic tissue velocity $(r=0.72, p<0.05)$ but showed no significant correlation with the segmental strain and strain rate. The time-to-maximal LA volume was positively correlated with the time-to-anterior and -posterior wall longitudinal peak strains $(\mathrm{r}=0.45, \mathrm{p}=0.04$ and $\mathrm{r}=0.52, \mathrm{p}=0.02$, respectively). The time-to-maximal LA volume was positively correlated with the time-to-longitudinal peak tissue tracking of the anterior wall $(\mathrm{r}=0.59, \mathrm{p}<0.01)$, posterior wall $(\mathrm{r}=0.48, \mathrm{p}=0.03)$, septum $(\mathrm{r}=0.63, \mathrm{p}<0.01)$, and lateral wall $(\mathrm{r}=0.58, \mathrm{p}=0.01)$, and with the timeto-circumferential peak tissue tracking $(r=0.49, \mathrm{p}=0.04)$. The LA active emptying fraction was positively correlated with the anterior wall longitudinal peak systolic tissue velocity $(r=0.51, p=0.02)$ and the posterior wall circumferential peak systolic tissue velocity $(r=0.68$, $\mathrm{p}<0.01)$ and the longitudinal peak late diastolic tissue velocity $(\mathrm{r}=0.54$, $\mathrm{p}=0.01)$. The LA active emptying fraction was positively correlated with the longitudinal peak late diastolic strain rates of the anterior $(r=0.49$ $\mathrm{p}=0.02$ ) and lateral walls ( $\mathrm{r}=0.49, \mathrm{p}=0.02)$.

In mitral regurgitation, it is shown that the circumferential and radial tissue velocities were significantly lower than the longitudinal tissue velocities. The maximal LA volumes were positively correlated with the LA filling velocities $(\mathrm{r}=0.70, \mathrm{p}<0.01)$ and with the radial peak systolic velocity $(r=0.53, p=0.03)$ but were not significantly correlated with the segmental late diastolic tissue velocity, strain, and strain rate. The minimal LA volume had a negative correlation with the LA active emptying fraction $(\mathrm{r}=-0.64, \mathrm{r}<0.01)$. The time-to-maximal LA 
Table 2: Doppler echocardiographic features of mitral valve

\begin{tabular}{llllllll}
\hline Parameters & MV-E & MV-A & MV-VTI & MV-A VTI & LA-MAX VOL & ATRIAL FRACTION & ELECTRO-MECH DELAY \\
\hline Valid & 43 & 43 & 43 & 43 & 43 & 43 & 43 \\
Mean & 2.45 & 1.97 & 81.81 & 27.21 & 91.08 & 1.16 & 538.70 \\
Standard deviation & 0.57 & 0.67 & 28.60 & 10.53 & 59.10 & 5.44 & 55.28 \\
\hline
\end{tabular}

MV: Mitral valve, MAX: Maximum, VOL: Volume

Table 3: Volumetric parameters of left atrium

\begin{tabular}{lllllll}
\hline Parameters & LA MAX VOL & LA MIN VOL & LA VOL_P & LA EXPANSION INDEX & $\begin{array}{l}\text { LA PASSIVE EMPTYING } \\
\text { FRACTION }\end{array}$ & $\begin{array}{l}\text { LA ACTIVE EMPTYING } \\
\text { FRACTION }\end{array}$ \\
\hline $\mathrm{N}$ (valid) & 43 & 43 & 43 & 43 & 43 & 43 \\
Mean & 91.08 & 50.54 & 66.51 & 128.35 & 29.30 & 31.40 \\
Standard deviation & 59.10 & 51.15 & 51.07 & 91.48 & 13.07 & 15.97 \\
\hline
\end{tabular}

LA: Left atrium, MAX: Maximum, VOL: Volume

Table 4: LA function indices

\begin{tabular}{lll}
\hline Parameters & Mitral annular area & Ejection force \\
\hline Valid & 43 & 43 \\
Mean & 8.43 & 9.32 \\
Standard deviation & 2.70 & 4.12 \\
\hline
\end{tabular}

LA: Left atrium

Table 5: Global LA strain

\begin{tabular}{ll}
\hline $\mathbf{N}$ (valid) & $\mathbf{4 3}$ \\
\hline Mean & 7.48 \\
Median & 5.20 \\
Standard deviation & 6.57 \\
Minimum & 0.20 \\
Maximum & 25.80 \\
\hline
\end{tabular}

Table 6: Comparison of LA global strain in groups with MR and without MR

\begin{tabular}{llll}
\hline MR & $\mathbf{N}$ & Mean \pm standard deviation & p value \\
\hline MR & 38 & $7.38 \pm 6.27$ & 0.79 \\
No MR & 5 & $8.22 \pm 9.42$ & \\
\hline
\end{tabular}

LA: Left atrium, MR: Magnetic resonance

Table 7: Comparison of LA function in groups with MR and without MR

\begin{tabular}{llll}
\hline MR & N & $\begin{array}{l}\text { Mean } \pm \text { standard } \\
\text { deviation }\end{array}$ & p value \\
& & & 0.3 \\
\hline $\begin{array}{l}\text { LA ACTIVE EMPTYING } \\
\text { FRACTION }\end{array}$ & & & \\
$\quad$ NO MR & 38 & $30.67 \pm 16.28$ & \\
$\quad$ MR & 5 & $36.96 \pm 13.53$ & 0.6 \\
LA PASSIVE EMPTYING & & & \\
FRACTION & & & \\
$\quad$ NO MR & 38 & $28.93 \pm 12.44$ & \\
$\quad$ MR & 5 & $32.14 \pm 18.73$ & 0.3 \\
ATRIAL_FRACTION & & & \\
$\quad$ NO MR & 38 & $1.27 \pm 5.78$ & 0.5 \\
$\quad$ MR & 5 & $0.38 \pm 0.10$ & \\
LA_EXPANSION INDEX & & & \\
$\quad$ NO MR & 38 & $125.40 \pm 88.91$ & \\
$\quad$ MR & 5 & $150.86 \pm 118.48$ & \\
\hline
\end{tabular}

LA: Left atrium, MR: Magnetic resonance

volume was positively correlated with the time-to-posterior and lateral wall longitudinal peak strain $(r=0.58, p=0.01$ and $r=0.51, p=0.04$, respectively), with the time-to-circumferential peak strain ( $\mathrm{r}=0.52$, $\mathrm{p}=0.04)$ and with the time-to-circumferential peak systolic velocity $(\mathrm{r}=0.52, \mathrm{p}=0.04)$. The time-to-maximal LA volume was positively correlated with the time-to-longitudinal peak tissue tracking of the posterior wall $(\mathrm{r}=0.66, \mathrm{p}<0.01)$ and septum $(\mathrm{r}=0.71, \mathrm{p}<0.01)$ and with the time-to-circumferential peak tissue tracking $(r=0.66, p<0.01)$. The LA active emptying fraction was negatively correlated with the lateral wall longitudinal peak late diastolic tissue velocity $(\mathrm{r}=-0.64, \mathrm{p}<0.01)$ and positively correlated with the lateral wall longitudinal peak strain $(\mathrm{r}=0.50, \mathrm{p}=0.03)$. The LA active emptying fraction was positively correlated with the posterior wall and lateral wall longitudinal peak late diastolic strain rate $(r=0.56, p=0.01$ and $r=0.67, p<0.01$, respectively) and with the radial peak late diastolic strain rate $(r=0.71, p<0.01)$.

Significant mitral valve disease leading to LA remodeling results in LA dysfunction that can be assessed by $2 \mathrm{D}$ indices of assessing LA function as well as LA strain. The LA active emptying fraction, which is the measure of contractility, is reduced in study participants. LA conduit function indicated by the LA passive emptying fraction is significantly reduced in both MS and MR patients. LA reservoir function is showed by the new echocardiographic parameter - LA strain is significantly reduced in mitral valve disease patients. However, in the literature, the studies have shown a significant reduction in such parameters in comparison with controls.

\section{CONCLUSION}

It is found that LA contractile, reservoir, and conduit function are significantly reduced in mitral valve diseases due to increased hemodynamic load. Echocardiographic features including 2D echo and tissue Doppler indices made LA function assessment possible, reliable, and highly reproducible. There is no significant different in the global LA strain among mitral valve disease patients regardless of etiology of the mitral valve diseases whether MR or MS.

\section{ACKNOWLEDGMENTS}

The authors would like to acknowledge the role of the Manipal University in encouraging us and allowing the use of their resources for the conduct of this study.

\section{REFERENCES}

1. Matsuda Y, Toma Y, Ogawa H, Matsuzaki M, Katayama K, Fujii T, et al. Importance of left atrial function in patients with myocardial infarction. Circulation 1983;67(3):566-71.

2. Hoit BD, Shao Y, Gabel M, Walsh RA. In vivo assessment of left atrial contractile performance in normal and pathological conditions using a time-varying elastance model. Circulation 1994;89(4):1829-38.

3. Otto CM, Bonow RO. Valvular heart disease. In: Libby P, Bonow RO, Mann DL, Zipes DP, Braunwald E, editors. Braunwald's Heart Disease: A Textbook of Cardiovascular Medicine. $8^{\text {th }}$ ed. Philadelphia, PA: Elsevier Publications; 2008.

4. Sirbu C, Herbots L, D'hooge J, Claus P, Marciniak A, Langeland T, 
et al. Feasibility of strain and strain rate imaging for the assessment of regional left atrial deformation: A study in normal subjects. Eur J Echocardiogr 2006;7(3):199-208

5. Tops LF, van der Wall EE, Schalij MJ, Bax JJ. Multi-modality imaging to assess left atrial size, anatomy and function. Heart 2007;93(11):1461-70.

6. Kircher B, Abbott JA, Pau S, Gould RG, Himelman RB, Higgins CB, et al. Left atrial volume determination by biplane two-dimensional echocardiography: Validation by cine computed tomography. Am Heart J 1991;121:864-71.

7. Matsuoka H, Hamada M, Honda T, Kobayashi T, Suzuki M, Ohtani T, et al. Measurement of cardiac chamber volumes by cine magnetic resonance imaging. Angiology 1993;44(4):321-7.

8. Marmor A, Frankel A, Blondeheim DS, Satinger A, Front D. Scintigraphic assessment of atrial function in patients with longstanding hypertension. Radiology 1984;151(2):483-6.

9. Pislaru C, Abraham TP, Belohlavek M. Strain and strain rate echocardiography. Curr Opin Cardiol 2002;17(5):443-54.

10. Wang T, Wang M, Fung JW, Yip GW, Zhang Y, Ho PP, et al. Atrial strain rate echocardiography can predict success or failure of cardioversion for atrial fibrillation: A combined transthoracic tissue Doppler and transoesophageal imaging study. Int J Cardiol 2007;114(2):202-9.

11. Kokubu N, Yuda S, Tsuchihashi K, Hashimoto A, Nakata T, Miura T, et al. Noninvasive assessment of left atrial function by strain rate imaging in patients with hypertension: A possible beneficial effect of renin-angiotensin system inhibition on left atrial function. Hypertens Res 2007;30(1):13-21.

12. Thomas L, McKay T, Byth K, Marwick TH. Abnormalities of left atrial function after cardioversion: An atrial strain rate study. Heart 2007;93(1):89-95

13. Merckx KL, De Vos CB, Palmans A, Habets J, Cheriex EC, Crijns HJ, et al. Atrial activation time determined by transthoracic Doppler tissue imaging can be used as an estimate of the total duration of atrial electrical activation. J Am Soc Echocardiogr 2005;18(9):940-4.

14. Cameli M, Caputo M, Mondillo S, Ballo P, Palmerini E, Lisi M, et al. Feasibility and reference values of left atrial longitudinal strain imaging by two-dimensional speckle tracking. Cardiovasc Ultrasound 2009;7(1):6 